\title{
Adaptive Frequency Hopping in Ad Hoc Networks with Rayleigh Fading and Imperfect Sensing
}

\author{
Ralph Tanbourgi, Jens P. Elsner, Holger Jäkel and Friedrich K. Jondral
}

\begin{abstract}
A probabilistic model for adaptive frequency hopping (AFH) based wireless ad hoc networks with Rayleigh fading, where interference is due to self- and to slow-varying external interference, is proposed. Different AFH sensing techniques are studied in terms of area spectral efficiency (ASE) and it is shown that self-interference can have a negative effect on the AFH mechanism. In particular, packet error rate and received signal strength sensing suffer when self-interference is high, while carrier detection based sensing remains robust. The proposed model further offers powerful means for system optimization.
\end{abstract}

Index Terms-Adaptive frequency hopping, ad hoc networks, area spectral efficiency.

\section{INTRODUCTION}

I $\mathrm{N}$ [1], a non-collaborative coexisting approach for wireless personal area networks (WPANs) termed adaptive frequency hopping (AFH) was proposed for systems operating in non-regulated spectrum. In $\mathrm{AFH}$, the hopping sequences are adapted to the (long-term) channel qualities such that bad channels, polluted by external interference created by other systems, are avoided. Channel sensing, required for detecting external interference, is typically based on received signal strength, packet error rate, carrier detection or a combination of them.

In view of the rapid growth of the number of applications for WPANs, the issue of self-interference has gained importance as well: Multiple WPANs using the same wireless technology will have to coexist in overlapping areas, e.g., in medical health-care environments [2] or with Bluetooth scatternets [3].

In such scenarios, performance is limited by both types of interference, without one type dominating the other [4]. As a result, the AFH sensing process becomes imperfect, i.e., the measurements contain not only external but also selfinterference. Hence, an AFH mechanism optimized without accounting for self-interference may loose its optimality in implementations, even resulting in an adverse behavior of $\mathrm{AFH}$ when self-interference increases.

In this letter, we develop a model that accounts for both types of interference. It is based on stochastic geometry, cf. [5]. We gain insights into the impact of self-interference on AFH by studying the area spectral efficiency (ASE).

\section{System Model}

We consider a slow FH-CDMA wireless network consisting of identical nodes ${ }^{1}$ distributed in $\mathbb{R}^{2}$ and having $m$ orthogonal

The authors are with the Karlsruhe Institute of Technology's (KIT's) Communications Engineering Lab (CEL), Karlsruhe, Germany. Email: $\{$ ralph.tanbourgi, jens.elsner, holger.jaekel, friedrich.jondral\}@kit.edu

${ }^{1}$ They do not necessarily have to share the same network topology, e.g., dense deployment of multiple WPAN picocells. frequency channels. Medium access is assumed to be slotted. To control the amount of self-interference, resulting from concurrent transmissions of nodes of the same type, a guard zone (GZ) mechanism is employed by the receivers, cf. [6]. Self-interference is fast-varying (on the order of a fixed packet duration) due to alternating transmitter/receiver $(\mathrm{Tx} / \mathrm{Rx})$ roles and possibly mobility. To capture these dynamics, we will use a probabilistic (spatial) model, cf. [5].

The nodes also experience external interference by a colocated system having the following characteristics: (i) the interference power levels $N_{1}, \ldots, N_{m}$ are node-wise identically distributed and channel-wise independent and identically distributed (i.i.d.) (ii) the realizations $n_{1}, \ldots, n_{m}$ are slowlyvarying (on the order of several transmissions). To counteract external interference, every node employs AFH to avoid bad channels with a common sensing threshold $\theta$. A node's set of active channels depends solely on its own view on the interference. The number of active channels for each node is lower bounded by $k$. We denote by $V \in[k, m]$ the total number of active channels in the network and assume that the (adapted) hopping sequences are always pseudo-random.

In a randomly chosen slot, some nodes independently decide to transmit (transmission attempts). The positions of these nodes are assumed to follow a stationary Poisson point process (PPP) of intensity $\lambda_{\mathrm{b}}$. Upon accessing the medium, each of these potential Txs tunes to a channel $\ell$ according to the (adapted) hopping sequence of the intended Rx situated at fixed (target) distance $d .{ }^{2}$ From the Displacement Theorem [8], it follows that the Rx positions follow a PPP with intensity $\lambda_{\mathrm{b}}$. Only those transmission attempts not inhibited by the GZ are carried out.

We focus on a probe link in channel $\ell$ with an $\mathrm{Rx}$ placed in the origin $o$ and an intended Tx placed $d>1$ units away at position $z \in \mathbb{R}^{2}$. We denote by $U \in[k, m]$ the number of active channels of the probe Rx. Since the two PPPs (Tx and $\mathrm{Rx}$ set) are both stationary, the probe link is typical for the network, cf. [5].

The far-field power path loss between two arbitrary positions $x, y$ is $\|x-y\|^{-\alpha}$, where $\alpha>2$ is the path loss exponent. ${ }^{3}$ We assume Rayleigh fading between the nodes, while for the channel from external source to the nodes we do not make any specific assumption. The power fading between a Tx at $x$ and an $\mathrm{Rx}$ at $y$ is denoted by the unit-mean exponentially distributed $G_{x y}$. The power fading in channel $\ell$ between external source and an $\operatorname{Rx}$ at $y$ is denoted by $H_{y}$.

\footnotetext{
${ }^{2}$ Allowing $d$ to vary does not affect the throughput significantly [7].

${ }^{3}$ Since the GZ mechanism inhibits close-by transmissions, we do not have to be concerned with near-field effects.
} 
Following [9], we embed the GZ mechanism into our model using a two step approach:

1) Large-scale density: First, the large-scale density, i.e., the density "seen from a distant viewpoint", of Txs in channel $\ell$ is derived using the fact that the Txs inhibited by the GZs of the Rxs follow a Matérn-like point process (cf. [9]). With this fact, the large-scale density in an active channel $\ell$, when $V=v$ channels are active in the network, is obtained by ${ }^{4}[8]$

$$
\lambda_{\ell}(v):=\frac{\lambda_{\mathrm{b}}}{v} \frac{1-e^{-C}}{C} .
$$

Note that $\lambda_{\ell}(v)=0$ for an inactive channel $\ell . C$ is the average number of Txs situated in the GZ of the probe Rx, contending for the same channel, and is given by

$$
C=\int_{\mathbb{R}^{2}} \frac{\lambda_{\mathrm{b}}}{v} e^{-\gamma\|x\|^{\alpha}} \mathrm{d} x=\frac{\lambda_{\mathrm{b}}}{v} \pi \Gamma\left(1+\frac{2}{\alpha}\right) \gamma^{-\frac{2}{\alpha}},
$$

where $\gamma$ is the GZ threshold and $\Gamma(a):=\int_{0}^{\infty} t^{a-1} e^{-t} \mathrm{~d} t$ is the Gamma function. Conditioned on $V=v$, the total largescale density in the network is $\lambda(v):=\sum_{\ell=1}^{m} \lambda_{\ell}(v)$. Thus, we have $\lambda_{\ell}(v)=\lambda(v) / v$ for every active channel. Throughout this work, we will write $\lambda(v) / v$ for the large-scale density in an active channel.

2) Shot-range inhibition: Secondly, the interference field at $o$ is modeled using a non-homogeneous PPP approximation capturing the shot-range inhibition. With this approximation, the Txs in channel $\ell$ around the probe $\mathrm{Rx}$ in $o$ (receiving in channel $\ell$ ) follow a PPP $\Phi_{\ell}:=\left\{x: x \in \mathbb{R}^{2}\right\}$ of intensity $\left(1-\exp \left(-\gamma\|x\|^{\alpha}\right)\right) \lambda_{\ell}(v)$. The term $1-\exp \left(-\gamma\|x\|^{\alpha}\right)$ can be seen as a position-dependent thinning probability, i.e., the probability of retaining a Tx at $x$ because it will not sense the GZ beacon from the probe $\mathrm{Rx}$ in $o,{ }^{5} \mathrm{cf}$. [9].

We assume that all nodes transmit with unit power. The signal-to-interference ratio (SIR) in channel $\ell$ at the probe $\mathrm{Rx}$ is given by ${ }^{6}$

$$
\operatorname{SIR}_{\ell}:=\frac{G_{z o} d^{-\alpha}}{H_{o} N_{\ell}+\sum_{\mathrm{x} \in \Phi_{\ell} \backslash\{z\}} G_{\mathrm{xo}}\|\mathrm{x}\|^{-\alpha}}=\frac{G_{z o}}{H_{o} \eta_{\ell}+Y_{\ell}},
$$

where $\eta_{\ell}:=N_{\ell} d^{\alpha}$ and $Y_{\ell}:=d^{\alpha} \sum_{\mathrm{x} \in \Phi_{\ell} \backslash\{z\}} G_{\mathrm{xo}}\|\mathrm{x}\|^{-\alpha}$ are the normalized external and self-interference power in $o$. The distributions of $\eta_{\ell}$ and $N_{\ell}$ are identical except for scaling.

\section{Area Spectral Efficiency - Perfect Sensing}

We now study the ASE with perfect sensing, i.e., when the sensing measurements contain only external interference.

\section{A. Performance metrics}

The outage probability (OP) of the probe link in channel $\ell$, given $V=v$ active channels in the network, is the probability that $\operatorname{SIR}_{\ell}$ is below a predefined threshold $\beta$, i.e.,

$$
q\left(\lambda_{\ell}(v), \eta_{\ell}\right):=\mathbb{P}^{! z}\left(\operatorname{SIR}_{\ell}<\beta\right) .
$$

\footnotetext{
${ }^{4}$ We assume that the density $\lambda_{\mathrm{b}}$ is "equally distributed" over all $v$ channels. Care will be taken such that this assumption always holds (see Definition 1+2).

${ }^{5}$ Alternatively, $\exp \left(-\gamma\|x\|^{\alpha}\right)$ can be seen as the probability that the $\mathrm{Rx}$ in $o$ will detect the transmission of a Tx at $x$ (receiver sensing).

${ }^{6}$ Interference is treated as white noise at the decoder. Thermal noise is neglected.
}

In this work, the ASE $\Upsilon$ is defined as the average aggregated per-channel large-scale density weighted by the probability of success of the probe link. The averaging is over the external interference, which influences the large-scale density through $V$ on the one hand, and the OP of the probe link through $\eta_{1}, \ldots, \eta_{m}$ on the other, i.e.,

$$
\Upsilon:=\mathbb{E}_{V, \eta_{1}, \ldots, \eta_{m}}\left[\sum_{\ell=1}^{m} \lambda_{\ell}(V)\left(1-q\left(\lambda_{\ell}(V), \eta_{\ell}\right)\right)\right] .
$$

\section{B. Analysis}

The ASE will strongly depend on how the nodes are affected by external interference: If the nodes form close-by piconets and the external system is far away, most nodes will experience the same external interference and, thus will avoid the same channels. In contrast, if the nodes have an independent view on the external interference, their sets of active channels are i.i.d. In this case, all channels will remain active from the network's viewpoint with equal load. We will consider these two extreme cases - full dependence and mutual independence - for the analysis, yielding lower and upper performance bounds. ${ }^{7}$

Definition 1 (Full dependence (FD) scenario). In the FD scenario all nodes observe the same realization $n_{1}, \ldots, n_{m}$ and will thus discard the same channels. Consequently, the set of active channels (of the probe receiver) is the same for all nodes, implying $V \equiv U$. The large-scale density in each active channel is therefore $\lambda_{\ell}(U)=\lambda(U) / U$.

Definition 2 (Mutual independence (MI) scenario). In the MI scenario, every node sees its own realization $n_{1}, \ldots, n_{m}$. Thus, the sets of active channels are independent among the nodes. Since the $N_{1}, \ldots, N_{m}$ are i.i.d., the load in each channel should be equal, implying $V \equiv m$. As a result, the large-scale density is $\lambda_{\ell}(m)=\lambda(m) / m$ in each channel.

We first calculate the OP at the probe $\mathrm{Rx}$ in $o$ :

Lemma 1. The OP of the probe link in an active channel $\ell$ conditioned on the fact that $V=v$ channels are active is

$$
q\left(\lambda_{\ell}(v), \eta_{\ell}\right):=1-\mathcal{L}_{H_{o}}\left(\beta \eta_{\ell}\right) \Omega(v),
$$

where $\mathcal{L}_{H_{o}}(\cdot)$ is the Laplace transform of $H_{o}$,

$$
\Omega(v):=\exp \left[\frac{-2 \pi^{2} \lambda(v) s^{\frac{2}{\alpha}}}{v \alpha \sin \frac{2 \pi}{\alpha}}\left(1-e^{\gamma s} \frac{\Gamma\left(1-\frac{2}{\alpha}, \gamma s\right)}{\Gamma\left(1-\frac{2}{\alpha}\right)}\right)\right],
$$

and $s:=\beta d^{\alpha} . \Gamma(a, b):=\int_{b}^{\infty} t^{a-1} e^{-t} \mathrm{~d} t$ is the upper incomplete gamma function.

Proof: The OP of the probe link is calculated as

$$
\mathbb{P}^{! z}\left(\operatorname{SIR}_{\ell}<\beta\right)=1-\mathbb{E}_{\Phi_{\ell}}\left[\exp \left(-\beta\left(\eta_{\ell}+Y_{\ell}\right)\right)\right],
$$

which is computed using the Laplace functional of a PPP [8]. The equality follows from the PPP approximation, Slivnyak's Theorem [8] and conditioning to $\Phi_{\ell}$.

Using this Lemma, we can derive the ASE:

\footnotetext{
${ }^{7}$ The modeling of a more realistic semi-dependent scenario, where colocated nodes experience the same external interference and external interference decorrelates between largely separated nodes, is analytically intractable.
} 
Theorem 1. The ASE $\Upsilon$ for the FD scenario $(V \equiv U)$ with perfect sensing is

$$
\begin{aligned}
\Upsilon^{\mathrm{FD}}= & \lambda(k) \Omega(k) \int_{\theta}^{\infty} \mathbb{E}_{\eta}[\mathcal{L}(\beta \eta) \mid \eta \leq t] \mathrm{d} \mathbb{P}\left(\eta_{(k+1)}=t\right) \\
& +\mathbb{E}_{\eta}\left[\mathcal{L}_{H_{0}}(\beta \eta) \mid \eta \leq \theta\right] \sum_{u=k+1}^{m} \lambda(u) \Omega(u) \mathbb{P}(U=u),
\end{aligned}
$$

where $\eta_{(k+1)}$ is the $(k+1)$-th order statistic of $\eta_{1}, \ldots, \eta_{m}$ and $\mathbb{P}(U=u)$ is given in (13).

See Appendix A for the proof. With the above expression the ASE can be calculated numerically for all distributions of $\eta$. For $H_{o} \equiv 1$ (path loss only), $\mathcal{L}_{H_{o}}(\beta \eta)=e^{-\beta \eta}$.

Corollary 1. The ASE $\Upsilon$ for the MI scenario $(V=m)$ with perfect sensing is

$$
\begin{aligned}
\Upsilon^{\mathrm{MI}}= & \lambda(m) \Omega(m)\left[\int_{\theta}^{\infty} \mathbb{E}_{\eta}[\mathcal{L}(\beta \eta) \mid \eta \leq t] \mathrm{d} \mathbb{P}\left(\eta_{(k+1)}=t\right)\right. \\
& \left.+\mathbb{E}_{\eta}\left[\mathcal{L}_{H_{0}}(\beta \eta) \mid \eta \leq \theta\right](\mathbb{P}(U \leq m)-\mathbb{P}(U=k))\right] .(10)
\end{aligned}
$$

Proof: The proof is analogous to that of Theorem 1.

\section{Area Spectral Efficiency - Imperfect Sensing}

We now study the ASE of AFH with imperfect sensing, i.e., when the measurements contain both external and selfinterference, for three commonly used sensing schemes. To obtain long-term observations, the measurements are typically averaged over time. For analytical tractability we assume that the averaging period is sufficiently large so that all fluctuations (self-interference and fading) vanish completely.

1) Received signal strength (RSS): RSS measurements can be used to detect a co-located system. By measuring the RSS when being idle, a node can determine the channel qualities. When normalized to the average received power, the measurement $\eta_{\ell}+\mathbb{E}\left[Y_{\ell}\right]$ is compared to a threshold $\theta_{\mathrm{r}}$.

2) Packet error rate (PER): This method implicitly measures the channel qualities by estimating the PER and comparing it to a threshold $\theta_{\mathrm{p}}$. With a sufficiently large averaging period, the measurement will converge to the OP from (6).

3) Carrier detection $(C D)$ : $\mathrm{CD}$ can be used to robustly detect signals from external systems. Here, it is assumed that a node performs the sensing process when it is in idle mode. Since the signal to be detected is superimposed by other signals from the network, the detection process is successful only if the ratio $\frac{\eta_{\ell}}{\mathbb{E}\left[Y_{\ell}\right]}$ is above a predefined threshold $\theta_{\mathrm{c}}$.

Lemma 2. The normalized average self-interference measured in an active channel, conditioned on $V=v$, is given by

$$
\bar{Y}(v):=\frac{2 \pi \lambda(v) d^{\alpha}}{v(\alpha-2)} \gamma^{1-\frac{2}{\alpha}} \Gamma\left(\frac{2}{\alpha}\right) .
$$

Proof: The Lemma is obtained by writing

$$
\bar{Y}(v)=d^{\alpha} \int_{\mathbb{R}^{2}} \mathbb{E}\left[G_{x o}\right]\|x\|^{-\alpha}\left(1-e^{-\gamma\|x\|^{\alpha}}\right) \frac{\lambda(v)}{v} \mathrm{~d} x
$$

and changing to polar coordinates. The equation follows from Slivnyak's and Campbell's Theorem, cf. [8].

We now can compute the ASE with imperfect sensing:

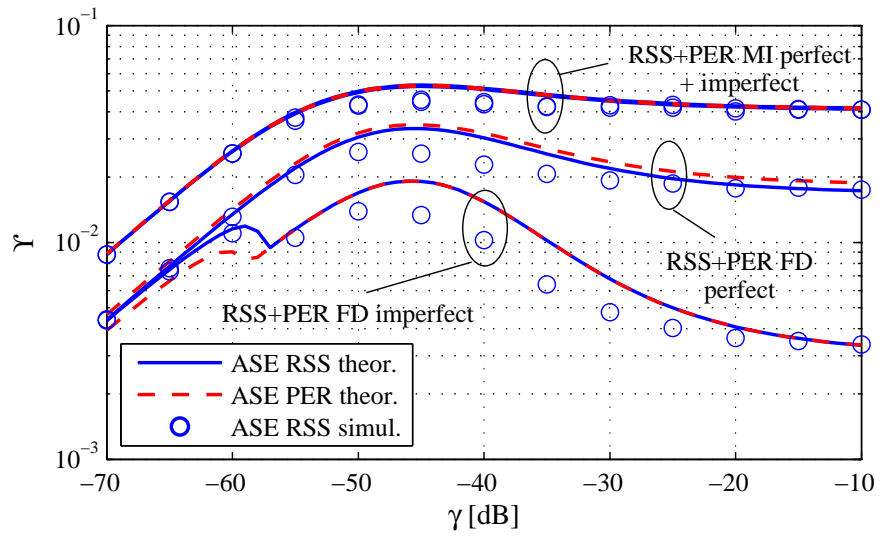

Fig. 1. ASE $\Upsilon$ vs. GZ threshold $\gamma$. The marks indicate the simulation results for RSS obtained without the PPP approximation.

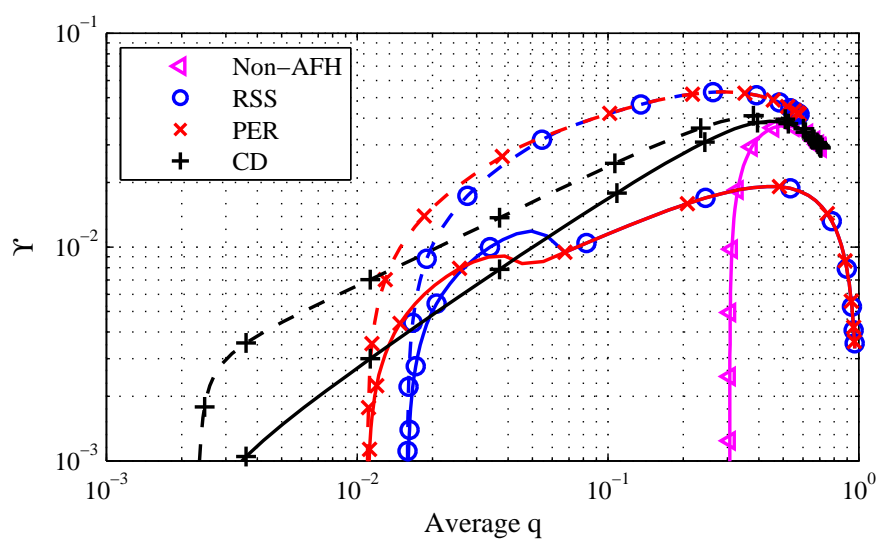

Fig. 2. ASE $\Upsilon$ vs. average OP $q$. Solid line (-) and dashed line (- -) indicate the FD and the MI scenario, respectively.

Corollary 2. The ASE from Theorem 1 (respectively Corollary 1) with imperfect sensing can be calculated by setting $\theta \equiv \theta_{\mathrm{r}}-\bar{Y}(v)$ for RSS, $\theta \equiv \frac{1}{\beta} \log \frac{\Omega(v)}{1-\theta_{\mathrm{p}}}$ for PER and $\theta \equiv \theta_{\mathrm{c}} \bar{Y}(v)$ for $\mathrm{CD}$.

The new thresholds are obtained by (theoretically) isolating external from self-interference in the measurement, resulting in a shift of the original thresholds. Note that the new thresholds now depend on $v$ through $\bar{Y}(v)$ (RSS, CD) and $\Omega(v)$ (PER).

\section{NUMERICAL EXAMPLES}

For the numerical examples, the following parameters were used: $\lambda_{\mathrm{b}}=0.1, \alpha=4, \beta=2, d=10, m=79, k=20$, $\theta_{\mathrm{r}}=-14 \mathrm{~dB}, \theta_{\mathrm{p}}=5 \%, \theta_{\mathrm{c}}=12 \mathrm{~dB}$. The $\eta_{1}, \ldots, \eta_{M}$ are log-normally distributed with $\mu=-14 \mathrm{~dB}$ and $\sigma=16 \mathrm{~dB}$. No fading between external source and the nodes is assumed. Fig. 1 shows the ASE vs. the GZ threshold $\gamma$ for RSS and PER sensing. In this example, for the FD scenario the ASE with imperfect sensing is considerably lower than for perfect sensing, particularly for large $\gamma$. This is because selfinterference increases the background interference level and hence, causes the AFH mechanism to remove channels which in turn reduces the interference avoidance feature of FH. This effect can be well observed between $-60 \leq \beta_{c} \leq-55$, where self-interference unnecessarily starts to trigger the $\mathrm{AFH}$ 
mechanism which results in an ASE drop. Note that the analytical values deviate from the simulation results by a small amount which is due to the Poisson approximation in the GZ model. This deviation, however, is observed only in a limited region, since for low $\gamma$ and/or $\lambda_{\mathrm{b}}$ the approximation is valid, while for large $\gamma$ the GZ inhibition effect vanishes. The latter can be seen by analyzing (1) and (2), showing that the medium access strategy converges to slotted Aloha as $\gamma$ increases [9].

Fig. 2 shows the ASE vs. the average OP $q$ (through varying $\gamma$ ) with imperfect sensing. It can be seen that AFH yields large improvements compared to non-AFH in terms of ASE and OP: Only by avoiding bad channels, OPs less than 0.3 are possible in this case. For high $\lambda_{\mathrm{b}}$ and high $\gamma$ (self-interferencelimited regime), RSS and PER exhibit poor performance. In contrast, $\mathrm{CD}$ has the desirable feature that external interference can no longer be detected when self-interference is high. As a result, all $m$ channels remain active and self-interference is effectively avoided.

\section{CONCLUSION}

We have proposed a model for analyzing AFH similar to [1] for dense ad hoc networks. In such networks, selfinterference has a great impact on the AFH sensing process. It was found that both RSS and PER sensing yield low ASE when self-interference is strong, since with these two methods frequency hopping is performed on less bandwidth. CD can overcome this problem because external interference will no longer be detected when self-interference becomes dominant. To increase robustness of AFH, we used the GZ threshold to control the impact of self-interference on $\mathrm{AFH}$, thereby increasing ASE. Using stochastic geometry to model the node positions has the advantage of averaging over all possible topologies (spatial averaging), thus enabling the study of AFH in ad hoc networks in a unifying way. This model may be of interest for the design of AFH-based ad hoc networks: For example, one could optimize the ASE over the GZ threshold $\gamma$ and the AFH threshold $\theta$ subject to an outage constraint, thus optimally balancing self- and external interference.

\section{ACKNOWLEDGEMENTS}

The authors gratefully acknowledge that their work is partially supported within the priority program 1397 "COIN" under grant No. JO 258/21-1 by the German Research Foundation (DFG).

\section{APPENDIX A}

\section{PROOF OF THEOREM 1}

Let $\eta_{(1)}, \ldots, \eta_{(m)}$ be the order statistics of the $\eta_{\ell}$, i.e., $\eta_{(\ell)} \leq \eta_{(\ell+1)}$ for all $1 \leq \ell<m$. Then,

$$
\mathbb{P}(U=u)= \begin{cases}\mathbb{P}\left(\eta_{(k+1)}>\theta\right), & u=k \\ \mathbb{P}\left(\eta_{(u)} \leq \theta, \eta_{(u+1)}>\theta\right), & k<u<m \\ \mathbb{P}\left(\eta_{(m)} \leq \theta\right), & u=m\end{cases}
$$

is true. Moreover, the $\eta_{\ell}$ are identical for every node in the FD scenario, so only $V \equiv U \in[k, m]$ channels will be active (cf. Definition 1). Without loss of generality, we assume that the channels $1, \ldots, U$ are the active ones. Replacing the upper limit in the sum by $U$ and conditioning (5) on $U=u$ yields

$$
\begin{aligned}
\Upsilon^{\mathrm{FD}} & =\mathbb{E}_{U}\left[\sum_{\ell=1}^{u} \mathbb{E}_{\eta_{(\ell)}}\left[\lambda_{\ell}(u)\left(1-q\left(\lambda_{\ell}(u), \eta_{\ell}\right) \mid U=u\right]\right]\right. \\
& =\mathbb{E}_{U}\left[\sum_{\ell=1}^{u} \lambda_{\ell}(u) \mathbb{E}_{\eta_{(\ell)}}\left[\left(1-q\left(\lambda_{\ell}(u), \eta_{\ell}\right) \mid U=u\right]\right]\right. \\
& =\mathbb{E}_{U}\left[u \lambda_{\ell}(u)\left(1-\mathbb{E}_{\eta}\left[q\left(\lambda_{\ell}(u), \eta_{\ell}\right) \mid U=u\right]\right)\right] \\
& =\mathbb{E}_{U}\left[\lambda(u)\left(1-\mathbb{E}_{\eta}\left[q\left(\lambda(u) / u, \eta_{\ell}\right) \mid U=u\right]\right)\right] .
\end{aligned}
$$

(15) follows from the independence of $\lambda_{\ell}(u)$ and $\eta_{\ell}$ given $U=$ $u$. (16) follows from the fact that, conditioned on $U=u$, the $\eta_{\ell}$ of the active channels, after removing their ordering, are i.i.d. with truncated distribution (cf. [10, p. 17, Theorem 2.5]) which reduces the sum to a $u$-fold expression. Note that $\lambda_{\ell}(u)$ is the same for all active channels. (17) follows from $\lambda(u)=u \lambda_{\ell}(u)$, which makes the $q$ of the active channels depend only on the i.i.d. $\eta_{\ell}$ of the active channels. Next, we rewrite the outer expectation in the form $\mathbb{E}[f(X)]=\sum_{i} f\left(x_{i}\right) \mathbb{P}\left(X=x_{i}\right),{ }^{8}$ and we consider the $f\left(x_{i}\right) \mathbb{P}\left(X=x_{i}\right)$ terms:

1) Case $u=k$ : In this case, $\eta_{(k+1)}>\theta$ and by (13), we have $\mathbb{P}(\eta \leq \cdot \mid U=k)=\mathbb{P}\left(\eta \leq \cdot \mid \eta \leq \eta_{(k+1)}\right)$ for all (unordered) active channels. Hence, the first term yields

$$
\lambda(k) \Omega(k) \int_{\theta}^{\infty} \mathbb{E}_{\eta}[\mathcal{L}(\beta \eta) \mid \eta \leq t] \mathrm{d} \mathbb{P}\left(\eta_{(k+1)}=t\right) .
$$

2) Case $k<u \leq m$ : Similarly, the $\eta$ of the (unordered) active channels conditioned on $U=u$ have truncated distribution $\mathbb{P}(\eta \leq \cdot \mid U=u)=\mathbb{P}\left(\eta \leq \cdot \mid \eta_{(u)} \leq \theta, \eta_{(u+1)}>\theta\right)=$ $\mathbb{P}(\eta \leq \cdot \mid \eta \leq \theta)$. Therefore, the remaining terms are

$\lambda(u) \Omega(u) \mathbb{E}_{\eta}\left[\mathcal{L}_{H_{o}}(\beta \eta) \mid \eta \leq \theta\right] \mathbb{P}(U=u), \quad k<u \leq m$,

where $\mathbb{P}(U=u)$ is given by (13).

\section{REFERENCES}

[1] "Part 15.2: Coexistence of wireless personal area networks with other wireless devices operating in unlicensed frequency bands," IEEE Std 802.15.2-2003, 2003.

[2] A. Soomro and D. Cavalcanti, "Opportunities and challenges in using wpan and wlan technologies in medical environments," IEEE Commun. Magazine, vol. 45, no. 2, pp. 114 -122, Feb. 2007.

[3] R. Kapoor, M. Sanadidi, and M. Gerla, "An analysis of bluetooth scatternet topologies," in Proc. of IEEE Conf. on Commun. (ICC), May 2003, vol. 1, May 2003, pp. $266-270$.

[4] C. D. M. Condeiro and D. P. Agrawal, Ad Hoc \& Sensor Networks: Theory And Applications. World Scientific Pub. Co. Inc., 2006.

[5] S. Weber, J. Andrews, and N. Jindal, "An overview of the transmission capacity of wireless networks," IEEE Trans. Commun., Dec 2010.

[6] A. Hasan and J. Andrews, "The guard zone in wireless ad hoc networks," IEEE Trans. Wireless Commun., Mar 2007.

[7] M. Kaynia, G. Oien, N. Jindal, and D. Gesbert, "Comparative performance evaluation of mac protocols in ad hoc networks with bandwidth partitioning," in IEEE 19th Int. Symposium on Personal, Indoor and Mobile Radio Comm. (PIMRC), Sep. 2008.

[8] F. Baccelli and B. Blaszczyszyn, "Stochastic geometry and wireless networks, volume 1+2: Theory and applications," Foundations and Trends in Networking, 2009.

[9] A. Hunter, R. Ganti, and J. Andrews, "Transmission capacity of multiantenna ad hoc networks with csma," in Forty Fourth Asilomar Conf. on Signals, Systems and Computers (ASILOMAR), Nov. 2010.

[10] H. A. David and H. N. Nagaraja, Order Statistics, 3rd ed. WileyInterscience, 2003.

${ }^{8}$ For the FD scenario, the thresholds in Corollary 2 depend on $U$. A normalization of (13) is therefore necessary such that $\sum_{u=k}^{m} \mathbb{P}(U=u)=1$. 